\title{
SYNERGISTIC ACTIVITY BETWEEN PRIMARY VISUAL NEURONS
}

\author{
Vishal Bharmauria $^{1}$, Lyes Bachatene ${ }^{1}$, Sarah Cattan ${ }^{1}$, Jean Rouat ${ }^{1,2}$, Stéphane Molotchnikoff ${ }^{1,2}$ \\ ${ }^{1}$ Dept. Sci. Biologiques, Univ. De Montreal, Montreal, QC, Canada; ${ }^{2}$ Univ. de Sherbrooke, \\ Sherbrooke, QC, Canada \\ Corresponding author: \\ Dr. Stéphane Molotchnikoff \\ Department of Biological Sciences \\ University of Montreal \\ C.P. 6128, succursale Centre-ville \\ Montréal QC, Canada, H3C 3J7 \\ Email: stephane.molotchnikoff@umontreal.ca
}

\begin{abstract}
:
Cortical microcircuitry plays a pivotal role in encoding sensory information reaching the cortex. However, the fundamental knowledge concerning the mechanisms that govern feature-encoding by these sub-networks is still sparse. Here, we show through multielectrode recordings in $\mathrm{V} 1$ of conventionally prepared anesthetized cats, that an avalanche of synergistic neural activity occurs between functionally connected neurons in a cellassembly in response to the presented stimulus. The results specifically show that once the reference neuron spikes in a connected neuron-pair, it facilitates the response of its companion (target) neuron for $50 \mathrm{~ms}$ and, thereafter, the excitability of the target neuron declines. On the other hand, the functionally unconnected neurons do not facilitate each other's activity within the $50 \mathrm{~ms}$ time-window. The added excitation (facilitation) of connected neurons is almost four times the responsiveness of unconnected neurons. This suggests that connectedness confers the added excitability to neurons; consequently leading
\end{abstract}




\section{to feature-encoding within the emergent 50 ms-period. Furthermore, the facilitation significantly decreases as a function of orientation selectivity spread.}

\section{Key Words: inter-neural relationships, primary visual cortex, functional network, microcircuit, synergy \\ INTRODUCTION}

Cortical neurons propagate information about the experience (input) through the complex temporal relationships of their action potentials (Kohn and Smith, 2005; Alloway and Roy, 2002;

Fujisawa et al., 2008; Barthó et al., 2004; Yoshimura et al., 2005; Samonds et al., 2006; Ghisovan et al., 2008). Neurons principally coordinate in local sub-networks termed 'cell-assemblies' to represent distinct cognitive entities (Ko et al., 2013; Ko et al., 2011; Perin et al., 2011; Kampa et al., 2011; Hebb,1949; Buzsaki, 2010). Visual cortex is perhaps the most studied area of the brain; yet, how local circuits emerge to encode specific information in response to a visual input, is a question to be deeply explored — that is, how the intriguing interplay between the stimulus and a microcircuit is engineered and modulated?

Rate coding and temporal coding seem to dominate the literature on population coding in response to a stimulating feature (Perkel and Bullock, 1968; Ratté et al., 2013; Ainsworth et al., 2012), and both appear to generate a code either as independent or mutually inclusive events (Ratté et al., 2013; Ainsworth et al., 2012). However, recently, authors (Ainsworth et al., 2012) have suggested a bias toward a precise temporal code (stimulus-driven-synchrony) that is a reflection of the anatomical architecture and synaptic biophysical properties of neurons (Ratté et al., 2013; Ainsworth et al., 2012). Synchronized neural activity implying specific functional connectivity has already been associated to the generation of coherent percepts (Fujisawa et al., 2008; Barthó et al., 2004; Ghisovan et al., 2008; Duret et al., 2006; Gray and Singer, 1989). 
Cross-correlogram function is an efficient tool to study synchrony between the neural spike trains (Perkel at al., 1967; Konig, 1995; Fujisawa et al., 2008; Barthó et al., 2004). Employing crosscorrelograms, we have already shown that co-active neurons in a V1 (layer II/III) cell-assembly frame a specific functional connectome in relation to a stimulus (Bretzner et al., 2001;

Bharmauria et al., 2012; Duret et al., 2006). In other words, how a cell-assembly waxes and wanes as the visual stimulus varies. It is to be mentioned that these functional connections were revealed within a $\pm 5 \mathrm{~ms}$ quasi-synchrony window in the cross-correlogram.

Building on the previous work, the goal of our current investigation is to observe and compare the most likely avalanche of neural activity between functionally connected, unconnected and untuned neuron-pairs in an assembly on a broader time-scale. That is, for how long, in relation to the spiking of one neuron, the discharge of the target neuron is modulated. We report that in V1 (layer II-III) of conventionally prepared anesthetized cats, the target neuron's discharge augments for $50 \mathrm{~ms}$ after reference neuron's spiking, and thereafter, the firing rate decreases. This is applicable to neurons (paired) that share orientation preference, as well as pairs exhibiting large difference in orientation tunings. On the other hand, no significant increase was noticed for unconnected and untuned cell-pairs within the same time-window. Thus, our results indicate that a functional network in V1 is set within $50 \mathrm{~ms}$ as a result of maximal synergistic activity, after the onset of the activity of companion cells in the assembly.

\section{EXPERIMENTAL PROCEDURES}

\section{Ethical approval}

Nine cats (Felis catus) were prepared in a conventional fashion for electrophysiological recordings in the primary visual cortex, as per the guidelines of Canadian Council on Animal care 
and approved by the Institutional Animal care and Use committee of Université de Montreal. The experiments were conducted in accordance with the Guide for Care and Use of Laboratory Animals of the National Institutes of Health (USA).

\section{Animal preparation}

Briefly, animals were sedated with acepromazine maleate (Atravet, Wyeth-Ayerst, Guelph, ON, Canada; 1 mg. $\mathrm{kg}^{-1}$, intramuscular) and atropine sulphate (ATRO-SA, Rafter, Calgary, AB, Canada; 0.04 mg. $\mathrm{kg}^{-1}$, intramuscular), and anesthetized with ketamine hydrochloride (Rogarsetic, Pfizer, Kirkland, QC, Canada; $25 \mathrm{mg} \cdot \mathrm{kg}^{-1}$, intramuscular). The cats were then paralyzed with 40 mg and maintained with $10 \mathrm{mg} \cdot \mathrm{kg}^{-1} \cdot \mathrm{h}^{-1}$ gallamine triethiodide (Flaxedil, Sigma Chemical, St. Louis, MO, USA; intravenous) administered in 5\% dextrose lactated Ringer's nutritive solution. General anesthesia was maintained by artificial ventilation with a mixture of $\mathrm{N}_{2} \mathrm{O} / \mathrm{O} 2$ (70:30) supplemented with $0.5 \%$ isoflurane (AErrane, Baxter, Toronto, ON, Canada). Electroencephalogram, electrocardiogram, rectal temperature and end-tidal $\mathrm{CO}_{2}$ partial pressure were monitored throughout the experiment, and kept in physiological ranges. Pupils were dilated with atropine, and Plano lenses with artificial pupils (5 mm diameter) were fixed. The electroencephalogram pattern ensured that the animals were properly anesthetized. The loci of the area centrales were inferred from the position of the blind spots. At the end of the experiment, the cats were euthanized intravenously with Sodium Pentobarbital (CEVA, Sante Animale). The injecting dose was $1 \mathrm{ml} / 2 \mathrm{~kg}$ of animal weight.

\section{Visual stimulation}

Monocular stimulation was done. The multi-unit receptive fields (RF) were mapped as the minimum response field (Barlow et al., 1967) by using a hand-held ophthalmoscope after clearly 
detectable activity was obtained. These preliminary tests revealed qualitative properties such as dimensions, velocity, orientation and direction selectivity. Visual stimuli were generated with a VSG 2/5 graphic board (Cambridge Research Systems, Rochester, England) and displayed on a 21-inch monitor (Sony GDM-F520 Trinitron, Tokyo, Japan) placed $57 \mathrm{~cm}$ from the cat's eyes, with $1024 \times 768$ pixels, running at $100-\mathrm{Hz}$ frame refresh. Stimuli were sine-wave drifting gratings covering the excitatory RF of neurons at both electrode tips. Contrast was set at $80 \%$. Mean luminance was $40 \mathrm{Cd} . \mathrm{m}^{-2}$. Optimal spatial and temporal frequencies were set at 0.24 cycles.deg ${ }^{-1}$ and 1.0-2.0 Hz range respectively, where V1 neurons are driven maximally by sinewave drifting gratings (Bardy et al., 2006).

\section{Electrophysiological recording and single-unit selection from multi-unit activity}

Multi-unit activity in the primary visual cortex was recorded at $410-820 \mu$ apart by a tungsten multi-electrode (Frederick Haer \& Co, Matrix Electrode). Recordings were performed in the supragranular layers (cortical depth $<1000 \mu \mathrm{m}$; mean $=650 \mu \mathrm{m}$ ). The signal from the microelectrodes was amplified, band-pass filtered $(300 \mathrm{~Hz}-3 \mathrm{kHz})$, digitized and recorded with $0.05 \mathrm{~ms}$ temporal resolution (Spike2, CED, Cambridge, England). All cells were discriminated on the basis of three criteria: 1) the spike-waveform difference, 2) principal component analysis (PCA) showing well dissociated clusters, and, 3) auto-correlograms showing no events at central point (Fujisawa et al., 2008; Barthó et al., 2004; Csicsvari et al., 1998; Harris et al., 2001). The stability of each cell's activity across conditions was verified qualitatively by visual control of the disposition of the clusters and the shapes of the waveforms.

The cluster analysis was performed using Spike2, CED, Cambridge, England in a 3-dimensional plot. The isolation distance was calculated as the Mahalanobis distance. Mahalanobis distance is 
the distance from the center of the cluster within which as many events belong to the other clusters as many belong to the specified cluster (Harris et al., 2001). In other words, given multivariate data values for which the values in each variable are normally distributed around a mean, this measure allows us to define boundaries of constant probability around the multidimensional centre of the distribution. This estimation allows the separation of a cluster from the nearest cluster. Units within Mahalanobis distance of 2.5 were considered for analysis.

Fig. 1 illustrates the protocol and isolation of neurons from a single electrode tip. Fig. 1A exemplifies the experimental protocol. Each drifting grating was presented 25 times lasting $180 \mathrm{~s}$, with varying inter-stimulus (1-3 s) intervals. Each trial lasted 4 s. Orientations of gratings were presented in a random order. Fig. 1B depicts the separated and averaged waveforms of four units recorded simultaneously. Each waveform is distinct, well separated, and corroborated by Principal Component Analysis. Fig. 1C shows the respective auto-correlograms of the four separated neurons, and no event at zero (corresponding to the refractory period of neuron) ascertains the individuality of the neuron. Fig. 1D illustrates the cluster analysis as explained above.

\section{Data analysis}

Once single cells were sorted out off-line from multi-unit spike trains, orientation tuning curves of cells were obtained from raw data and fitted with the von Mises function (Swindale, 1998).

$M(\theta)=A \cdot e^{b(\cos (\theta-c))}+\mathrm{d}$

where ' $A$ ' is the value of the function at the preferred orientation ' $c$ ', and ' $b$ ' is the width parameter. An additional parameter, 'd', represents the spontaneous firing rate of the cell. $M(\theta)$ is the firing rate of the neuron at orientation, ' $\theta$ '. This allowed us to determine with precision the 
preferred orientation of every cell. An orientation selectivity index (OSI) was calculated to ensure the tuning of neurons. It was measured using the fitted tuning curves by dividing the firing rate at baseline (orthogonal orientations) by the firing rate at the preferred orientation, and subtracting the result from one (Bachatene et al., 2013; Liao et al., 2004; Ramoa et al., 2001). The closer the OSI is to one, the stronger the orientation selectivity. Neurons with OSI $\geq 0.7$ were selected for further analysis.

\section{Cross-correlograms and network-formation}

Cross-correlograms were constructed between the spike trains of all the possible neuron-pairs at all the applied orientations to reveal the functional connections. We employed the shuffling minus subtraction algorithm based on the firing of the neurons, and it was subtracted from the raw cross-correlograms, which withdrew the evoked firing (Perkel et al., 1967). A significant peak of 2 ms (two adjacent $1 \mathrm{~ms}$ bins) or at least one significant bin (Alloway and Roy, 2002) was searched within a window of $\pm 5 \mathrm{~ms}$ around zero in the shift-corrected cross-correlograms to reveal a functional connection between two neurons. The statistical threshold for the significant peak was set at $95 \%$ (red curved line in cross-correlogram), and the probability (P) of the neuron firing in a bin is calculated as follows (Abeles, 1982):

Considering the spike train is a Poisson train, the probability $(\mathrm{P})$ of the neuron to fire in the small bin of the size $b$ is:

$\mathrm{P}=\mathrm{F}^{*} \mathrm{~b}$ and $\mathrm{F}=\mathrm{N} / \mathrm{T}$

where, ' $\mathrm{F}$ ' is the neuron frequency, ' $\mathrm{T}$ ' is the total time interval, and ' $\mathrm{N}$ ' is the number of spikes in that interval. 
The confidence limit in the cross-correlogram was calculated as per Abeles 1982:

The expected bin count $(\mathrm{C})$ for the histogram is:

$\mathrm{C}=\mathrm{P}^{*} \mathrm{NRef}$, where NRef is the number of the reference events.

The confidence limits for ' $\mathrm{C}$ ' are calculated using the assumption that $\mathrm{C}$ has a Poisson distribution.

Assuming that a random variable ' $\mathrm{S}$ ' has a Poisson distribution with parameter ' $\mathrm{C}$ ', then the $95 \%$ confidence limit is calculated as follows:

Low Conf. $=\mathrm{x}$ such that $\operatorname{Prob}(\mathrm{S}<\mathrm{x})=0.005$

High Conf. $=\mathrm{y}$ such that $\operatorname{Prob}(\mathrm{S}>\mathrm{y})=0.005$

In case when two cells recorded from the same tip fired in synchrony, their respective waveforms might sum up and consequently the shape of the resultant waveform is rejected as it falls outside the range of the template. In the present investigation 'synchrony' of spikes from the same tip is excluded from the analysis. We considered only spikes that fell beyond $\pm 1 \mathrm{~ms}$ after the central point in the cross-correlogram to make significant projections.

In addition, 'cumulative sums based on all histograms' of the firing rates indicated that the reference spike modulates the discharges of the target neuron. For every cross-correlogram the cumulative distribution of spikes was computed (it is shown above each cross-correlogram). This spike-triggered increase further determines the functional connectivity between both cells since the activity of the target cell depends upon the activity of the companion unit (reference neuron).

The cumulative sum (cs) graphs above histograms are calculated as:

If we have a histogram with bin counts bc $[i], i=1, \ldots, N$.

then the cumulative sum $\operatorname{cs}[\mathrm{i}]$ for a bin is:

for bin $1: \operatorname{cs}[1]=b c[1]-A$

for bin 2: $\operatorname{cs}[2]=b c[1]+b c[2]-A * 2$ 
for bin 3: $c s[3]=b c[1]+b c[2]+b c[3]-A * 3$, etc.

\section{Calculation of the response-modulation}

The 'response-modulation' following the spike of the reference cell is computed as a ratio. The ratio was calculated by considering the firing rates (counts/bin) derived from peristimulus time histograms (PSTH) of involved neurons. Fig. 2 illustrates the calculation of response-modulation (R) for two neurons. Fig. 2A corresponds to the raster plot of the blue neuron, whereas Fig. 2B represents the raster plot of the red neuron. The spikes of one neuron (blue) were set as reference (spike-by-spike analysis) and the activity of second neuron (red) was calculated in relation to the reference neuron. This computation leads to the histogram in Fig. $2 \mathrm{C}$. Thereafter, typically, several time-windows were considered for calculation of response-modulation. The first window is of $50 \mathrm{~ms}$ before reference spikes (zero mark), and second up to one second after the zero mark. This latter timescale was divided into $50 \mathrm{~ms}$ epochs to calculate the evolution of excitability modulation during the time-course of response up to one second after the zero mark. Thus, the ratio $(\mathrm{R})$ is calculated as:

$\mathrm{R}=\mathrm{Ni} / \mathrm{Nt}$

where, $\mathrm{Ni}=$ Average response of target neuron after zero (after the reference neuron spiked) in each $50 \mathrm{~ms}$ epoch, and $\mathrm{Nt}=$ Average response of target neuron within $50 \mathrm{~ms}$ before the zero mark, that is, prior to spiking of the reference cell. Fig. 2D depicts the excitability modulation of the target cell in the respective time-windows. N1/Nt represents the modulated activity of the red neuron with respect to the blue neuron in the first $50 \mathrm{~ms}$.

\section{RESULTS}


The main goal of the current investigation is to examine the time-course of synergistic crossinfluence between two simultaneously recorded neurons when one of them fires. In current analysis, 363 pairs were investigated from 39 recording sites (each site corresponds to recording from an electrode tip). Table 1 summarizes the statistics for analyzed neuron-pairs. Out of analyzed pairs, 165 were connected; 120 were unconnected and 78 were untuned. In the context of this paper, it is emphasized that simultaneously recorded neurons from a tip (see Fig. 1) are termed a 'cell-assembly'. Unconnected neuron-pairs were also investigated from the same cellassemblies. The untuned neurons-pairs were analyzed from other five recordings. It is to be mentioned that neurons did not belong to more than one assembly.

\section{Functional network revealed by cross-correlograms}

The first objective was to reveal the functional links between neurons in an assembly. Fig. 3A illustrates the shift-corrected cross-correlograms (see methods for details) based on which the functional network was disclosed between five simultaneously recorded neurons from a tip as shown in Fig. 3B. The first cross-correlogram represents the spike-triggered modulation of the green cell's (target, cell 2) activity in relation to the spiking of blue neuron (reference, cell 1). The cross-correlogram significantly peaks within $5 \mathrm{~ms}$ after zero, implying that cell 1 functionally projects onto cell 2 (unidirectional connection). The probability (p) of the significant peak in the cross-correlogram is 0.023 (see methods). This relation can be interpreted as a putative excitatory connection between the neurons (blue cell excites the green cell in the circuit). In addition, cumulative curve (shown above the cross-correlogram) of the firing rate shows the increased excitation of cell 2 almost immediately after cell 1 produced an action potential. This increased excitation further ascertains the functional link between the neurons. Similarly, cell 1 excites cell $4(\mathrm{p}=0.011)$ within the assembly. Cell 2 and cell 3 are reciprocally connected 
(bidirectional connection), that is, firstly cell 2 excites cell $3(\mathrm{p}=0.023)$ and after a brief period of inhibition characterized by a trough at zero in the histogram, cell 3 back-projects onto cell 2 (p $=0.014)$. The cross-correlogram between cell 5 and cell 2 is not significant, thus, no functional connection is inferred between them. The blue arrow depicts the direction of projection from one neuron to another.

\section{Synergistic activity within an assembly}

The analysis in Fig 3A, B disclosed the functional connections in a short quasi-synchrony ( $\pm 5 \mathrm{~ms}$ time-window) in cross-correlograms, and these links are indicative of putative and relatively direct synaptic links between the involved neurons. The next goal was to observe on a broader time-scale the evolution of the target cells' firing in the connected cell-pairs. To this aim, the PSTH's of neurons were compared to notice the modulation of the target cell's firing in relation to reference cell's spiking until one second (see methods, Fig. 2 for details). A typical example of a connected and an unconnected neuron pair is shown in Fig. 3C. As revealed in Fig. 3A, cell 4 (red) receives a connection from cell 1 while cell 3 (cyan) is unconnected to cell 1 (blue). Quite interestingly, in response to the same stimulus, the evoked firing (red curve) of cell 4 is considerably higher shortly after cell 1 has produced an action potential at zero mark. On the contrary, cell 1 failed to increase the evoked transient firing (blue curve) of cell 3, although the same stimulus was applied.

Fig. 3D displays the response-modulation histograms of the targets cells of the same cell-pairs as shown in Fig. 3A. The first histogram (Fig. 3D1) illustrates the firing modulation of cell 2 (target) in relation to the spiking of cell 1 . An increase of $82 \%$ neural activity is noticed in the first $50 \mathrm{~ms}$ after the reference cell firing. This suggests the response-facilitation of cell 2 by cell 1 
and advocates the synergistic coupling between them within this period. In a similar fashion, cell 1 promotes the neural discharge of cell 4 (Fig. 3D2) by $71 \%$ within $50 \mathrm{~ms}$, and thereafter, the facilitation subsides gradually. Cell 2 and cell 3 are reciprocally connected to each other (see Fig. 3A). Cell 2 increases the activity of cell 3 by $78 \%$ in the same period (Fig. 3D3); however, there is no facilitation of cell 2 by cell 3 (Fig. 3D4) suggesting a unidirectional facilitatory influence. In addition, it may be worthwhile to notice that there is an increase of the target neuron's firing at different epochs with respect to the trigger (reference) spikes. For example, cell 2 in relation to cell 1 exhibits augmented activity beyond $50 \mathrm{~ms}$ - a facilitation by $49 \%$ at $100 \mathrm{~ms}, 55 \%$ at 150 ms, a down-regulation of activity at $200 \mathrm{~ms}$, and again an increased activity corresponding to consequent bins. On the contrary, this does not apply to cell 1-cell 4 pair, and the facilitation is noticed only in the first $50 \mathrm{~ms}$. However, when the connected cell-pairs are summed up into a single unique assembly (Fig. 3D5), the average maximal synergistic effort (increase of activity by $47 \%$ ) was noticed within $50 \mathrm{~ms}$. Thus, although the magnitude-modulation of the firing of neurons within a cell-assembly varies considerably, a significant trend seems to apply to most cells: shortly following the spiking of the reference cell, the firing of the target cell increases for about $50 \mathrm{~ms}$ and, following this period, the responses decline; see next section.

\section{Difference between connected, unconnected and untuned units}

Thereafter, the facilitation-magnitudes were averaged and compared for connected, unconnected and untuned cell-pairs across all recorded clusters comprising all orientation spreads. Fig. 4 illustrates these results. Neurons in an assembly frame a specific microcircuit for a particular orientation or, generally, any exciting feature (Bretzner et al., 2001; Duret et al., 2006; Bharmauria et al., 2012). For closely tuned orientation groups $\left(\leq \pm 22^{\circ}\right)$, an optimal or nearoptimal orientation was chosen where most number of connections were found, since these 
orientations evoked the highest number of spikes. In case of assemblies with larger orientation spreads $\left(\geq \pm 22^{\circ}\right)$, a random orientation with highest number of connections was selected. In total, 165 connections were analyzed at all the selected orientations. Out of 165 connections, 111 were unidirectional and 27 were bidirectional/reciprocal (Table 1). It is to be mentioned that a bidirectional or reciprocal connection consists of two reverse projections (that is, two connections). Out of 165 connected pairs, a few pairs $(n=37)$ were analyzed at spontaneous activity, and the mean activity was found to be $0.87 \pm 0.11$ (Mean \pm SEM) in the 50 ms window, whereas, the same pairs exhibited a mean facilitation of $1.57 \pm 0.30$ upon stimulus presentation, that is, an increase of $57 \%$ in the chosen window. This suggests that stimulus presence leads to connections. Moreover, random unconnected neuron-pairs were also sampled $(n=120$, total unconnected pairs $=619$ ) across previously selected orientations. Additionally, untuned neuronpairs $(n=78)$ were also analyzed to compare the excitability with connected and unconnected cell-pairs.

The average facilitation for connected cell-pairs amounted to $46 \%$ (Fig. 4A) within the $50 \mathrm{~ms}$ time-window, whereas for unconnected cells the average increase amounted to $11 \%$ only (Fig. 4B). This spike-triggered facilitation in discharge rate for connected pairs might be attributed to the enhanced evoked discharges in response to the drifting grating which is present while cells' activities are being recorded. The difference in excitation between connected and unconnected units was significant (unpaired t-test, $\mathrm{p}<0.05$ ), suggesting that connectivity increases the excitability in a significant fashion. Lastly, the activity of untuned cell-pairs $(n=78)$ remained unchanged within the $50 \mathrm{~ms}$ window - a decrease in activity by $3 \%$ (Fig. 4C). In other words, the responsiveness of target cells in such cell-pairs was almost similar to the baseline firing. 
Furthermore, neuron-pairs were grouped into orientation-class spreads to determine the relation between the facilitation and orientation range of the neurons (Fig. 5). As described in the methods section, cells were tuned for orientation, and an orientation selectivity index (OSI) was calculated (Bachatene et al., 2013; Liao et al., 2004; Ramoa et al., 2001). The insert indicates the distribution of connections as a function of the orientation range of an assembly. The black part of the histogram corresponds to the number of connected pairs, whereas the grey part represents the number of unconnected pairs. The number above the histogram stands for the proportion of assemblies comprising that spread. The connections in all spreads are almost evenly distributed. This is attributed to a majority of closely tuned neurons (cell-pairs) that are present in all spreads. However, on a cell-pair basis, as expected, there is a decrease in firing facilitation within $50 \mathrm{~ms}$ as the orientation spread increased. The mean facilitation for close orientation spread $\left(44^{\circ}\right.$ or \pm $22^{\circ}$ ) was $1.53 \pm 0.13($ mean $\pm \mathrm{SEM})$, that is, an increase of $53 \%$ from the baseline firing. The values of this upsurge in neural activity were found to be $1.35 \pm 0.16$ and $1.32 \pm 0.13 \%$ respectively for $88^{\circ} / \pm 44^{\circ}(\mathrm{n}=24)$ and $132^{\circ} / \pm 66^{\circ}(\mathrm{n}=10)$ orientation ranges. It has to be mentioned that orientation spread beyond $132^{\circ}$ was not taken into account, as the neural selectivity (cell-pairs) comprising this spread approaches the opposite direction of the sine-wave drifting gratings. The regression analysis excluding the fourth (unconnected, UC) and fifth (untuned, UT) groups shows the decreasing trend of excitation as the spread increases $\left(\mathrm{R}^{2}=\right.$ 0.84). Interestingly, this result seems to indicate that as the orientation selectivity diverges, the cooperation between the units is maintained, even though the magnitude of the facilitation is weaker. In other words, a group of neurons in an assembly is more salient than other neurons within the assembly. There is a significant difference in excitability between cell-pairs in $44^{\circ}$ spread and unconnected units ( $\mathrm{p}<0.05$, unpaired t-test). 


\section{Significant cross-correlograms, decreased excitability and time-course of response-}

\section{modulation.}

Interestingly, a minority of cell-pairs displayed significant cross-correlograms (functional connections), but the respective target cells did not exhibit excitation within the $50 \mathrm{~ms}$ window of interest. Out of a total of 165 connected cell-pairs, 43 cells-pairs behaved in this fashion, that is, $26 \%$ of all the connected neuron-pairs. Fig. 6 illustrates a typical example. Fig. 6A shows a typical significant cross-correlogram for a connected neuron-pair. However, the activity of the same target cell is down-regulated by $19 \%$ within the $50 \mathrm{~ms}$ window, as evident from Fig. 6B.

The normalized and averaged added increase of excitability that may be attributed to inter-neural connectivity is computed in Fig. 7. The normalized mean activities of connected, unconnected and untuned cell-pairs were calculated for $200 \mathrm{~ms}$ (- $100 \mathrm{~ms}$ until $100 \mathrm{~ms}$ ). The computation produced three curves: the bold black curve indicates responses of cells that exhibited connections ( $\mathrm{n}=165)$; the gray plot represents the cells lacking functional connectivity $(\mathrm{n}=120)$; the dotted gray line stands for untuned pairs of cells $(n=78)$. Responses are aligned with the spikes of the reference cell at zero mark (vertical broken black line). The increase of cellular firing before zero mark is due to the presence of the stimulus in the receptive field as the stimulus is applied at the beginning of the trace. Thereafter, the transient evoked responses emerge, followed by a decline of excitation. Interestingly, for connected pairs, the transient response is enhanced by almost $400 \%$, whereas, there is a phasic increase of only $46 \%$ for unconnected pairs. Thus, there is almost 10 times significant (paired t-test for normalized responses, $\mathrm{p}<0.05$ ) increase (indicated by black arrow) in the activity of the connected neurons in comparison to the unconnected cell-pairs. Lastly, the activity of untuned neuron-pairs remains unmodulated as illustrated by the broken gray line. This spike-triggered difference in neural activity between 
connected and unconnected units underscores the avalanche of strong synergistic activity between connected cells.

\section{DISCUSSION}

In current investigation, the synergistic cooperation between connected, unconnected and untuned cell-pairs recorded in anesthetized cat V1 (layer II/III), was investigated and compared. We found that after the onset of the reference neuron's spike, the target neuron fires at a higher rate in connected cell-pairs for a brief period of $50 \mathrm{~ms}$, that is, the synergy is maximal within the $50 \mathrm{~ms}$ window, and thereafter this facilitation subsides. Secondly, the synergistic cross-influence decreases as a function of orientation spread.

\section{Methodological considerations}

Cellular excitation is certainly affected by anesthesia; however, it remained constant throughout the recording session. It has been demonstrated that spontaneous oscillations in anesthetized animals could induce correlations between neural firing (Poulet and Petersen, 2008). Likewise, varied oscillatory activities with different frequencies can be recorded in anesthetized animals (Gray and Singer, 1989; Gray et al., 1989). Previously, we have reported that connections were strongly related to a presented orientation; the pattern of cross-correlograms remained similar when a feature was presented repeatedly; and only a few connections ( $7 \%)$ were found at spontaneous activity. In addition, in current analysis, connected cell-pairs did not exhibit facilitation when stimulus was absent. Moreover, neurons that shared orientation preference exhibited more connections (Bharmauria et al. 2012). Furthermore, as the synergistic effect occurs within a relatively brief time-window, such short epoch does not support spontaneous 
modulation of excitability. Finally, targets were applied randomly. Collectively, these results suggest that connections are systematically established in response to a stimulus. One may argue that the increased activity for a brief period of time is simply due to co-activation of cells as the grating is presented, however, it is worthwhile to underline that the cross-correlations used to reveal the functional connections were shift-corrected to remove the stimulus evoked and locked components.

\section{Functional consequences}

We report that V1 (layer II/II) neurons impinge upon each other's activity for about $50 \mathrm{~ms}$ in response to a stimulus, and thereafter the facilitation declines. The connected neuron-pairs exhibit heightened activity in this time-window than unconnected pairs. This time-window coincides with previous reports (Maunsell and Gibson, 1992; Tovée and Rolls, 1994) wherein authors have postulated that majority of sensory information seems to be encoded in the first $50 \mathrm{~ms}$ of neural activity, and this time-period is enough to discriminate even the complex stimuli.

The foremost question arises as whether the target neurons in connected pairs receive an input from other recorded or unrecorded neurons in the circuit. Since a very few neurons were recorded from an electrode, it was not possible to quantify other unrecorded neurons in the circuit. However, from results obtained, we infer that the input of the reference neuron most likely causes the upsurge in the activity of the target neuron. In other words, the magnitude of excitation of the target cell is predominantly related to the activity of the reference unit; otherwise the target cells in unconnected pairs might have shown a pronounced augmented activity in $50 \mathrm{~ms}$, and as noticed, there is only $11 \%$ average facilitation in target cells of unconnected pairs in comparison to $46 \%$ for connected pairs. 
A few pairs exhibited functional connections despite a down-regulation of activity in the $50 \mathrm{~ms}$ time-bin. This can be related to the characteristic sparse activity in cortical circuits (Molotchnikoff and Rouat, 2011; Jayakumar et al., 2012; Barth and Poulet, 2012). Even though no increase of firing rate was noticed in the $50 \mathrm{~ms}$ bin, yet cross-correlating their neural spike trains yielded a significant cross-correlogram between neurons. This reflects the precise temporal locking (temporal code) between neural activities.

Certainly through a distributive system (Bernardet et al., 2002; Behrmann and Plautt, 2013), there are specific functional circuits dedicated to encoding processes. Such synergistic cross-influence between selected cells may assign specific roles and reduce multiple functions, hence, lessen ambiguities within the circuit. The augmented excitation for about $50 \mathrm{~ms}$ creates a 'window of opportunity' for downstream recipient assemblies to process the feature (Nadasdy, 2000; Wang et al., 2010). Such model would modulate the excitability of involved pathways allowing the increased transmission of information and obliteration of irrelevant inputs. In other words, within this framework, the stimulus-dependent saliency of a microcircuit is promoted.

Although the excitation decreased as a function of the orientation spread, nevertheless, it persisted. This finding reflects that, in an assembly, neurons that share tuning properties collaborate vigorously than neurons exhibiting dissimilar tunings. This seems to be in line with investigations reporting that V1 (layer II/III) neurons receive a diversity of oriented inputs from surrounding neurons, however, there is an enhanced connectivity between neurons that share orientation preference (Monier et al., 2003; Jia et al., 2010; Ko et al., 2011). Recently, we have shown that cell-assemblies develop heightened functional connections to a suitable orientation, and may wax and wane contingent upon the presented orientation (Ghisovan et al., 2008; Duret et 
al., 2006; Bharmauria et al., 2012; Bretzner et al., 2001). Then the results of the present investigation suggest that the formation of an encoding assembly of excited neurons may rest on two global processes: firstly, to an appropriate stimulus, there is a development of functional connections leading to construction of a functional connectome particular to that stimulus; secondly, these connections allow enhanced responses leading to relevant and salient neural activities that participate in signaling a specific target. That is, some cells in an assembly stand out since they exhibit augmented excitation in comparison to other cells. Collectively, these results seem to support recent reviews wherein authors have postulated that stimulus-specific information is encoded within salient sub-networks of neurons (Singer, 2013; Harris and MrsicFlogel, 2013). Thus, we construe from our results that the augmented neural activity (synergy) for particular neurons within an assembly frames the premise for the formation of a specific network in response to the presented stimulus.

We conclude that within a stimulus-encoding assembly in layer II/III of V1, the effect of one neuron on companion neuron(s) lasts $50 \mathrm{~ms}$, and thus the network is salient within this emergent period in relation to the stimulus. Our data also point to the fact that only a selected group of neurons within an assembly exhibits such increased excitation in response to a particular stimulus, while neighboring cells fail to show such transient surge of excitation. Thus, we suggest that any given stimulus discriminates the linked neurons that may encode its presence and properties.

\section{Acknowledgements}

The authors thank the anonymous referees for extensive and constructive reviews that helped improve the manuscript significantly. 


\section{Funding}

SM and JR were supported by CRSNG (Conseil de Recherches en Sciences Naturelles et en

Génie) FRQ-NT (Fonds de recherche du Québec - Nature et technologies).

\section{Author contributions}

VB did the experiments, analyzed the data and wrote the manuscript. LB and SC participated in experiments and analyses of data. JR contributed to the analyses of data. SM conceived the idea of study and contributed to data analyses and manuscript writing.

\section{Competing interests}

The authors declare no competing financial interests.

\section{REFERENCES}

Abeles M (1982) Quantification, smoothing, and confidence limits for single-units' histograms. J Neurosci Methods 5:317-325.

Ainsworth M, Lee S, Cunningham MO, Traub RD, Kopell NJ, Whittington MA (2012) Rates and rhythms: a synergistic view of frequency and temporal coding in neuronal networks. Neuron 75:572-583.

Alloway KD, Roy S (2002) Conditional cross-correlation analysis of thalamocortical neurotransmission. Behav Brain Res 135:191-196.

Bachatene L, Bharmauria V, Cattan S, Molotchnikoff S (2013) Fluoxetine and serotonin facilitate attractive-adaptation-induced orientation plasticity in adult cat visual cortex. Eur J Neurosci 38:2065-2077

Bardy C, Huang JY, Wang C, FitzGibbon T, Dreher B (2006) "Simplification" of responses of complex cells in cat striate cortex: suppressive surrounds and feedback inactivation. J Physiol 574:731-750.

Barlow HB, Blakemore C, Pettigrew JD (1967) The neural mechanism of binocular depth discrimination. J Physiol 193:327-342.

Barth AL, Poulet JF (2012) Experimental evidence for sparse firing in the neocortex. Trends Neurosci 35:345-355.

Barthó P, Hirase H, Monconduit L, Zugaro M, Harris KD, Buzsáki G (2004) Characterization of neocortical principal cells and interneurons by network interactions and extracellular features. J Neurophysiol 92:600-608. 
Behrmann M, Plaut DC (2013) Distributed circuits, not circumscribed centers, mediate visual recognition. Trends Cogn Sci 17:210-219.

Bernardet U, Verschure PF (2002) iqr: a tool for the construction of multi-level simulations of brain and behaviour. Neuroinformatics 8:113-134.

Bharmauria V, Bachatene L, Cattan S, Rouat J, Molotchnikoff S (2012) Network selectivity between primary visual neurons: Connectome Dynamics. Soc Neuros 572.07/FF8

Bretzner F, Aïtoubah J, Shumikhina S, Tan YF, Molotchnikoff S (2001) Modulation of the synchronization between cells in visual cortex by contextual targets. Eur J Neurosci 14:15391554.

Buzsáki G (2010) Neural syntax: cell assemblies, synapsembles, and readers. Neuron 68:362385.

Csicsvari J, Hirase H, Czurko A, Buzsáki G (1998) Reliability and state dependence of pyramidal cell-interneuron synapses in the hippocampus: an ensemble approach in the behaving rat. Neuron 21:179-189.

Duret F, Shumikhina S, Molotchnikoff S (2006) Neuron participation in a synchrony-encoding assembly. BMC Neurosci 7:72.

Fujisawa S, Amarasingham A, Harrison MT, Buzsáki G (2008) Behavior-dependent short-term assembly dynamics in the medial prefrontal cortex. Nat Neurosci 11:823-833.

Ghisovan N, Nemri A, Shumikhina S, Molotchnikoff S (2008) Synchrony between orientationselective neurons is modulated during adaptation-induced plasticity in cat visual cortex. BMC Neurosci 9:60.

Gray CM, Singer W (1989) Stimulus-specific neuronal oscillations in orientation columns of cat visual cortex. Proc Natl Acad Sci USA 86:1698-702.

Gray CM, König P, Engel AK, Singer W (1989) Oscillatory responses in cat visual cortex exhibit inter-columnar synchronization which reflects global stimulus properties. Nature 338:334-337.

Harris KD, Mrsic-Flogel TD (2013) Cortical connectivity and sensory coding. Nature 503:51-58.

Harris KD, Hirase H, Leinekugel X, Henze DA, Buzsáki G (2001) Temporal interaction between single spikes and complex spike bursts in hippocampal pyramidal cells. Neuron 32:141-149.

Hebb DO (1949) The organization of behavior. New York: John Wiley and Sons. 
Jayakumar J, Hu D, Vidyasagar TR (2012) Sparseness of coding in area 17 of the cat visual cortex: A comparison between pinwheel centres and orientation domains. Neuroscience 225:5564.

Jia H, Rochefort NL, Chen X, Konnerth A (2010) Dendritic organization of sensory input to cortical neurons in vivo. Nature 464:1307-12.

Kampa BM, Roth MM, Göbel W, Helmchen F (2011) Representation of visual scenes by local neuronal populations in layer 2/3 of mouse visual cortex. Front Neural Circuits 5:18.

Ko H, Cossell H, Baragli C, Antolik J, Clopath C, Hofer SB, Mrsic-Flogel TD (2013) The emergence of functional microcircuits in visual cortex. Nature 496:96-100.

Ko H, Hofer SB, Pichler B, Buchanan K, Sjöström PJ, Mrsic-Flogel TD (2011) Functional specificity of localsynaptic connections in neocortical networks. Nature 473:87-91.

Kohn A, Smith MA (2005) Stimulus dependence of neuronal correlation in primary visual cortex of the macaque. J Neurosci 25:3661-3773.

König P, Engel AK, Roelfsema PR, Singer W (1995) How precise is neuronal synchronization? Neural Comput 7:469-485.

Liao DS, Krahe TE, Prusky GT, Medina AE, Ramoa AS (2004) Recovery of cortical binocularity and orientation selectivity after the critical period for ocular dominance plasticity. J Neurophysiol 92:2113-2121.

Maunsell JHR, Gibson JR (1992)Visual response latencies in striate cortex of the macaque monkey. J Neurophysiol 68:1332-1344.

Molotchnikoff S, Rouat J (2011) Brain at work: time, sparseness and superposition principles. Front Biosc. 17:583-606.

Monier C, Chavane F, Baudot P, Graham LJ, Frégnac Y (2003) Orientation and direction selectivity of synaptic inputs in visual cortical neurons: a diversity of combinations produces spike tuning. Neuron 37:663-680.

Nádasdy Z (2000) Spike sequences and their consequences. J Physiol Paris 94:505-524.

Perin R, Berger TK, Markram H (2011) A synaptic organizing principle for cortical neuronal groups. Proc Natl Acad Sci USA 108:5419-5424.

Perkel DH, Gerstein GL, Moore GP (1967) Neuronal spike trains and stochastic point processes. I. The single spike train. Biophys J 7:391-418.

Perkel DH, Bullock TH (1968) Neural coding. Neurosci Res Prog Bull 6:221-348. 
Poulet JF, Petersen CCH (2008) Internal brain state regulates membrane potential synchrony in barrel cortex of behaving mice. Nature 454:881-885.

Ramoa AS, Mower AF, Liao D, Jafri SI (2001) Suppression of cortical receptor function prevents development of orientation selectivity in the primary visual cortex. J Neurosci 21:4299-4309

Ratté S, Hong S, De Schutter E, Prescott SA. (2013) Impact of neuronal properties on network coding: roles of spike initiation dynamics and robust synchrony transfer. Neuron 78:758-772.

Rolls ET, Tovée MJ (1994) Processing speed in the cerebral cortex, and the neurophysiology of backward masking. Proc R Soc Lond Biol 257:9-15.

Samonds JM, Zhou Z, Bernard MR, Bonds B (2006) Synchronous activity in cat visual cortex encodes collinear and cocircular contours. J Neurophysiol 95:2602-2616.

Singer W (2013) Cortical dynamics revisited. Trends Cogn Sci 17:616-626

Swindale NV (1998) Orientation tuning curves: empirical description and estimation of parameters. Biol Cybern 78:45-56.

Wang Q, Webber RM, Stanley GB (2010) Thalamic synchrony and the adaptive gating of information flow to cortex. Nat Neurosci 13:1534-1541.

Yoshimura Y, Dantzker JL, Callaway EM (2005) Excitatory cortical neurons form fine-scale functional networks. Nature 433:868-873. 


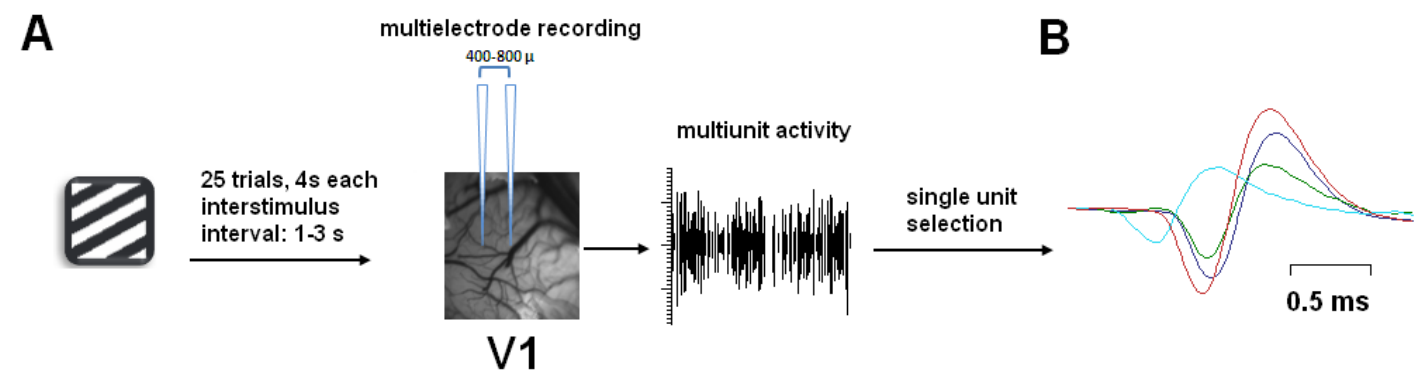

C
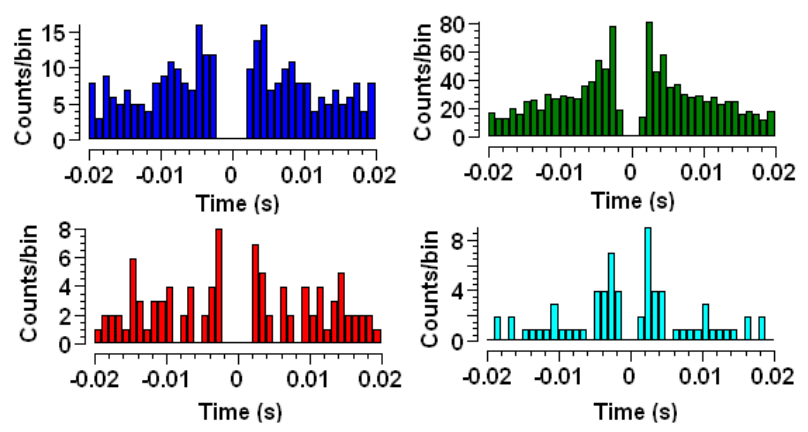

D

Fig. 1. Visual stimulation protocol and isolation of units. (A) Stimuli (drifting orientationgratings) were presented in a random order. Each stimulus was presented 25 times and each trial lasted 4 seconds with 1-3 ms inter-stimulus interval. Tungsten multi-electrodes (inter-electrode distance $=410 \mu$ ) were used to perform the multiunit recordings in layer II/III of V1 in cat. (B) Superimposed average waveforms of dissociated spikes from multi-unit recordings. (C) Autocorrelograms for the separated single units in (B). No event at zero representing the refractory period of neuron confirms the individuality of neuron. (D) Cluster analysis for the dissociated waveforms. The color scheme is respected in all figures. 
A

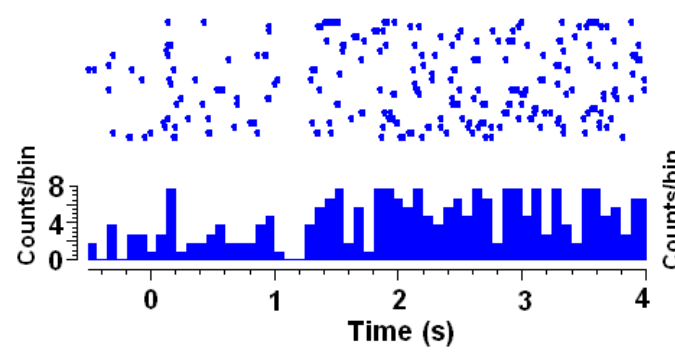

C

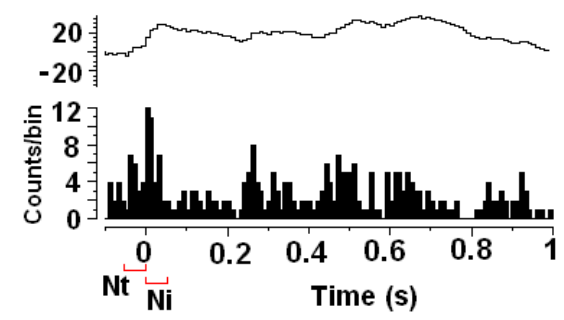

B

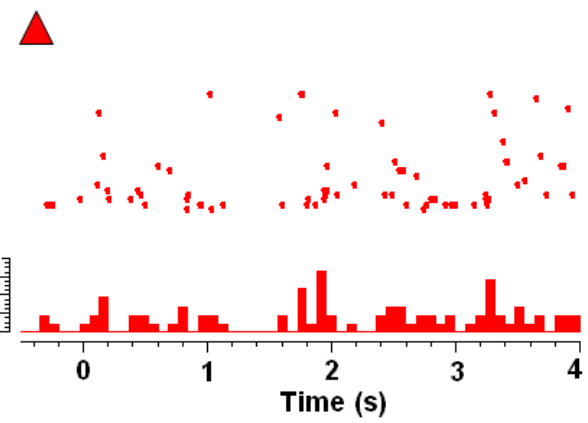

D

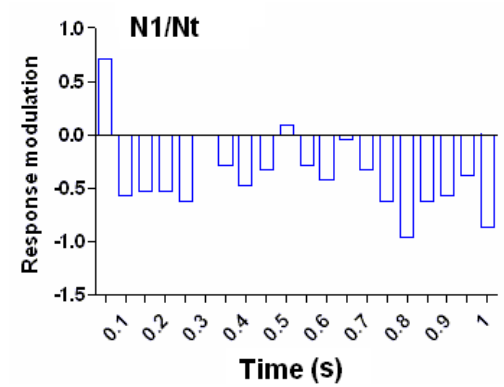

Fig. 2. Calculation of response-modulation. (A) Peristimulus time histogram (PSTH) of the reference neuron (blue) over 25 trials. (B) Peristimulus time histogram of the target neuron (red). (C) Histogram of the target neuron relative to the reference neuron spiking (spike-by-spike). (D) Response-modulation (R) histogram of the target neuron. 'Ni' stands for the mean response in every $50 \mathrm{~ms}$ bin after zero, whereas, ' $\mathrm{Nt}$ ' represents the average firing within $50 \mathrm{~ms}$ before zero mark. N1/Nt $(\mathrm{R})$ is the modulated response of the target neuron in the first $50 \mathrm{~ms}$ after zero. 


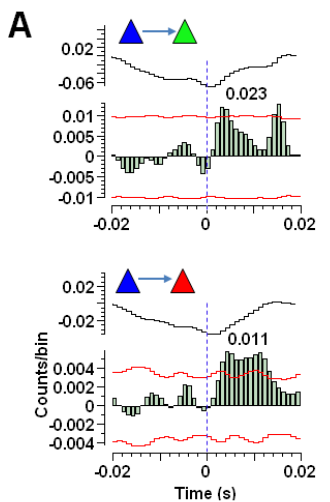

B

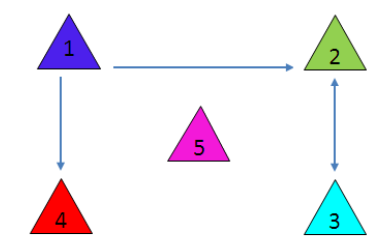

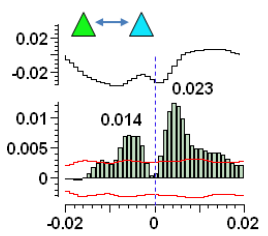
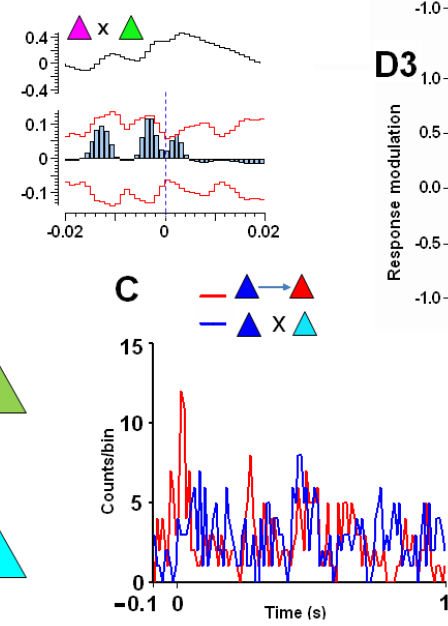
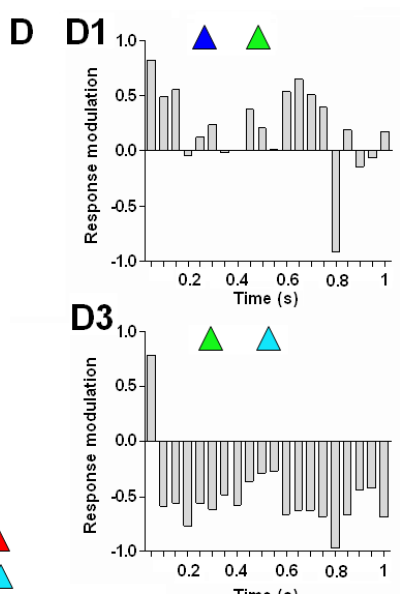

Time (s)
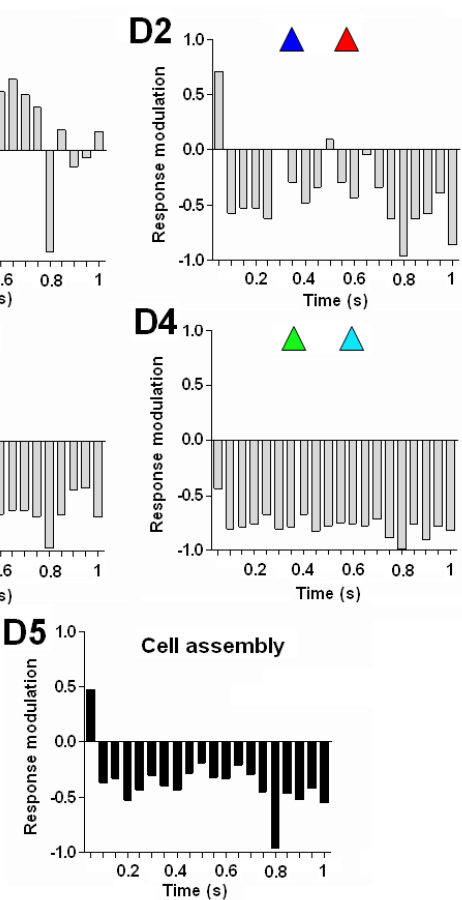

Fig. 3. Functional network and response-modulation in a cell-assembly. (A) Shift-corrected cross-correlograms to reveal the functional links between simultaneously recorded neurons shown in (B). The first cross-correlogram between blue cell (reference) and green cell (target) shows that target cell spikes within $5 \mathrm{~ms}$ once the reference cell fires and the probability of this discharge is 0.023 . This means that blue neuron projects onto green neuron and signifies the functional link between them. The red curved line represents the significance level (95\%). The curved line above the cross-correlogram corresponds to the cumulative sum based on histogram which further ascertains the functional link between neurons. The broken blue line depicts the zero in the cross-correlogram. The first neuron (colored triangle) in every cross-correlogram represents the reference neuron. (B) Functional network of simultaneously recorded neurons as disclosed from cross-correlogram analysis in (A). (C) A typical example of Peristimulus histogram comparison (PSTH) for neuron-pairs. Red curve stands for the connected pair whereas the blue curve depicts the unconnected pair. There is an augmented excitation of the target cell for connected cell-pair. (D) Response-modulation of target cells in cell-pairs shown in (A). Note the surge of activity in the first $50 \mathrm{~ms}$ for all the histograms (D1-D3) except (D4). (D5) Averaged histogram for a typical cell-assembly (D1-4). 
A Connected Cell-pairs

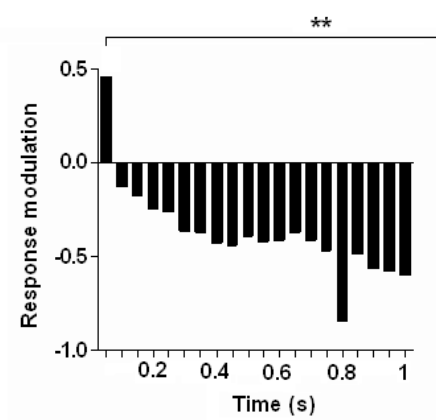

B Unconnected Cell-pairs

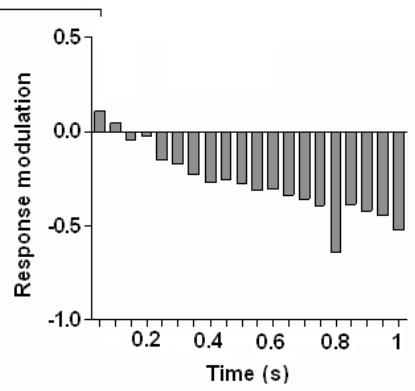

C Untuned Cell-pairs

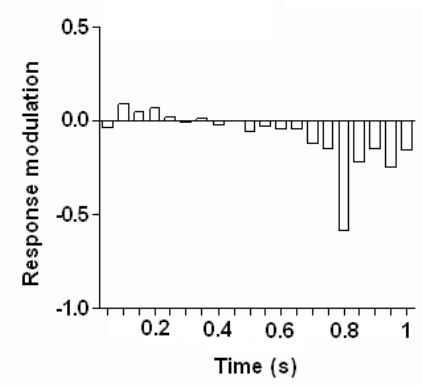

Fig. 4. Excitation comparison for connected, unconnected and untuned cell-pairs. (A)

Average response modulation for pooled in connected cells across all recordings. There is $46 \%$ facilitation in the first $50 \mathrm{~ms}$ epoch for the target cells in connected cell-pairs. (B) Average response modulation for all unconnected cell-pairs. The excitation is $11 \%$ in the $50 \mathrm{~ms}$ timewindow. There is a significant difference in facilitation for connected and unconnected units (unpaired t-test, $\mathrm{p}<0.05$ ). (C) Mean response modulation for untuned cell-pairs. The responses are down-regulated to $3 \%$, that is, the firing of target neurons remains similar to their baseline firing. 


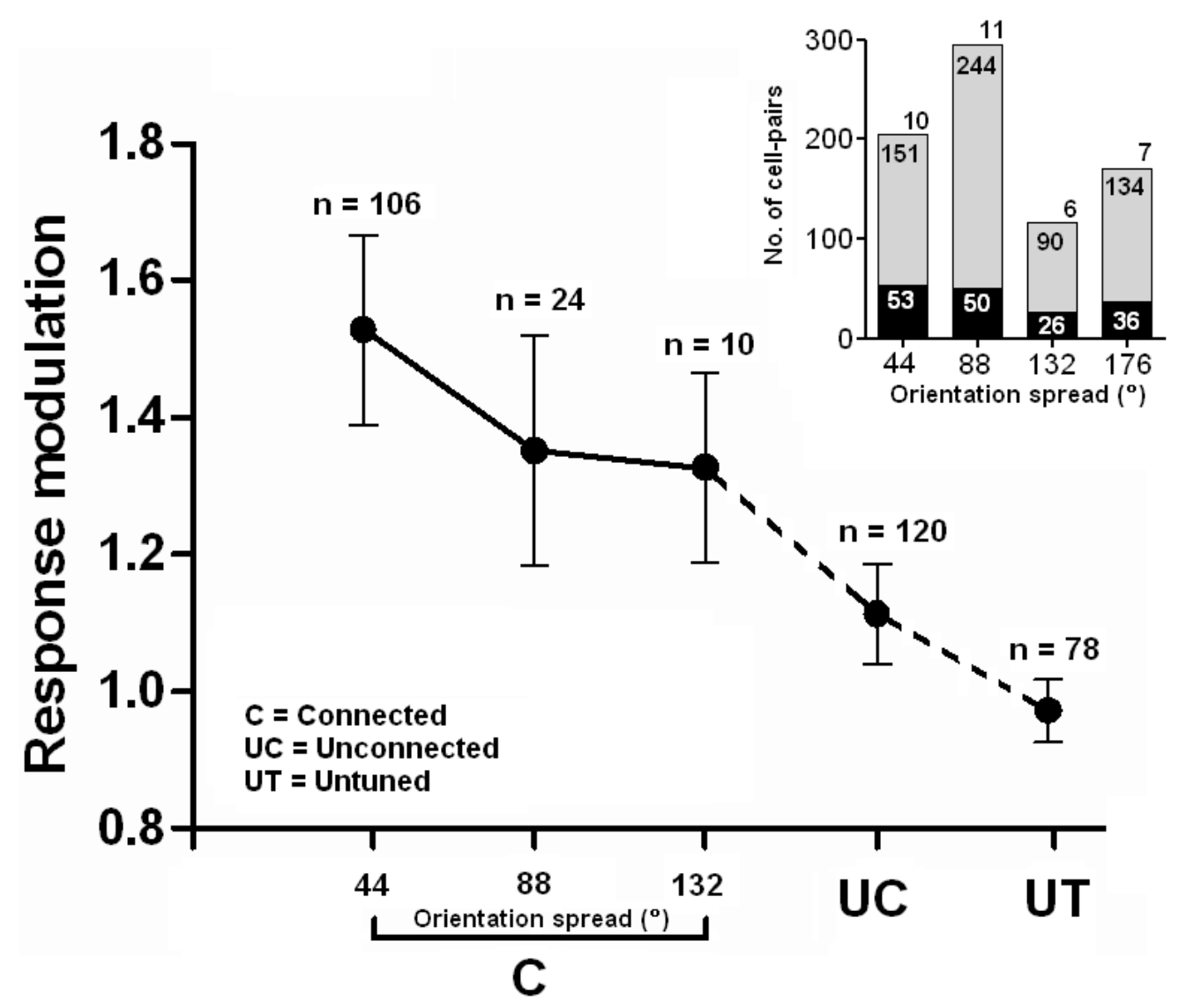

Fig. 5. Facilitation as a function of orientation spread. The first three points represent the connected (C) neuron-pairs in different orientation spreads. Linear regression analysis shows that there is a decrease in facilitation as the orientation range increases $\left(\mathrm{R}^{2}=0.84\right)$. The horizontal bars indicate the SEM. UC represents the unconnected cell-pairs, and UT corresponds to untuned cell-pairs. In general, the facilitation decreases from connected cell-pairs to untuned cell-pairs. Note: the last two dots (unconnected cells and untuned cells) are eliminated for regression analysis. Number of cell-pairs in each group is indicated by (n). The insert highlights the distribution of connected pairs as a function of the orientation range of an assembly. The black and grey part of histogram represents the number of connected and unconnected pairs respectively. The number above the histogram indicates the number of assemblies within the spread. 

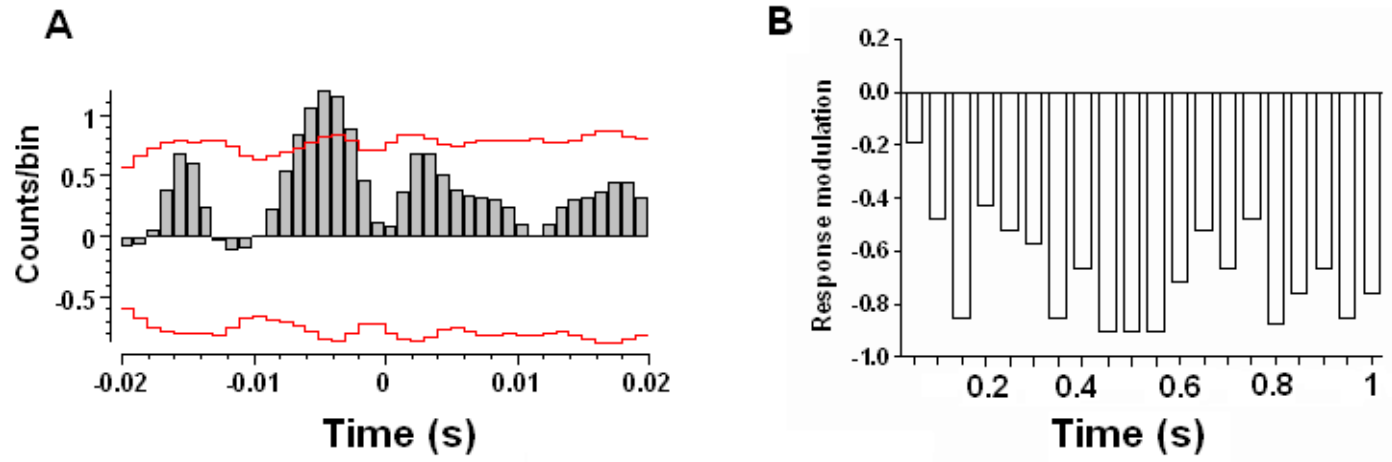

Fig. 6. A typical example of a cell-pair displaying a significant cross-correlogram (A), albeit, not exhibiting facilitation in the first $50 \mathrm{~ms}$ time-window (B). 


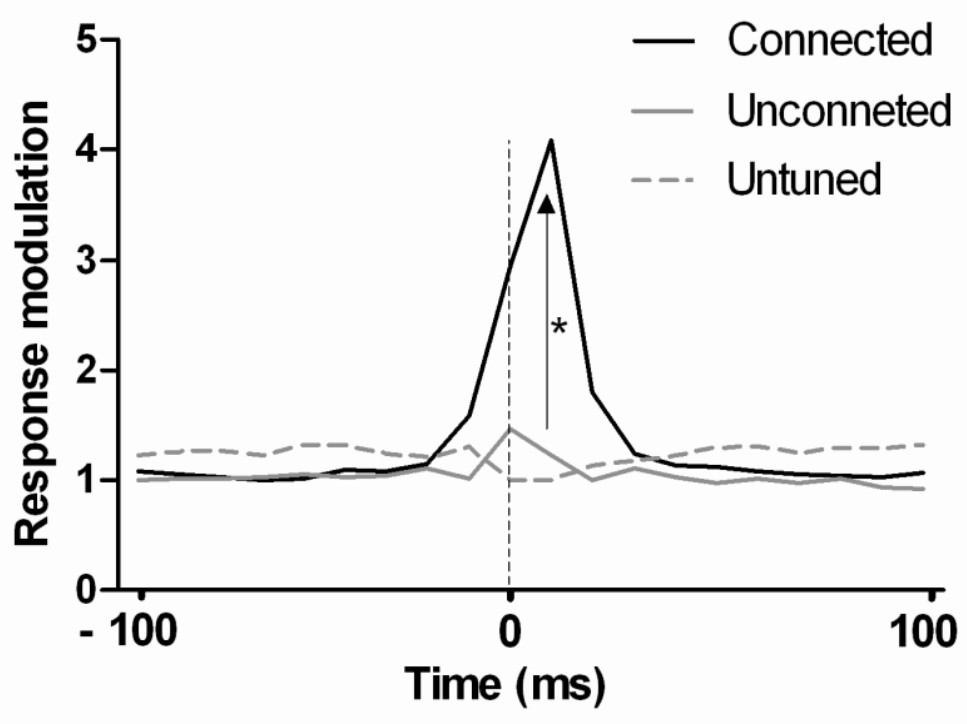

Fig. 7. Difference in excitation between connected, unconnected and untuned cell-pairs. Normalized facilitation curves for connected, unconnected and untuned cell-pairs. The black curve depicts the connected cells and shows a heightened activity for neurons in the $50 \mathrm{~ms}$ timewindow in comparison to unconnected (gray curve) and untuned (broken gray curve) neuronpairs. There is a significant increase (indicated by upright black arrow) in facilitatory activity for connected cell-pairs in comparison to unconnected pairs (asterisk represents the significance level, paired t-test for normalized responses, $\mathrm{p}<0.05$ ). The broken vertical black line indicates zero. 


\begin{tabular}{|c|c|c|c|}
\hline \multicolumn{4}{|c|}{ Total cell-assemblies (39 sites) } \\
\hline \multicolumn{3}{|c|}{$\begin{array}{l}\text { Tuned neurons ( } 34 \text { sites, } 178 \text { neurons) } \\
\text { Average neurons per cell-assembly: } \mathbf{5 . 2}\end{array}$} & $\begin{array}{l}\text { Untuned neurons ( } 5 \\
\text { sites, } 28 \text { neurons) } \\
\text { Average neurons per } \\
\text { cell-assembly: } \mathbf{5 . 6}\end{array}$ \\
\hline \multirow{2}{*}{\multicolumn{2}{|c|}{$\frac{\text { Connected pairs }}{\mathbf{1 6 5}}$}} & Unconnected pairs & \multirow{4}{*}{78} \\
\hline & & \multirow{3}{*}{ 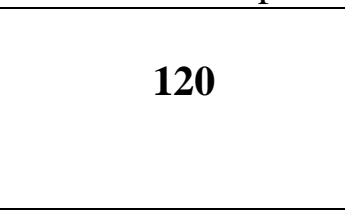 } & \\
\hline $\begin{array}{c}\text { Unidirectional } \\
\text { connections }\end{array}$ & $\begin{array}{c}\text { Bi-directional } \\
\text { connections }\end{array}$ & & \\
\hline 111 & 27 & & \\
\hline
\end{tabular}

Table 1. Assembly statistics for analyzed neuron-pairs. 